\title{
Comparisons and correlations of anti mullerian hormone and thyroid stimulating hormones in fertile and subfertile women
}

\author{
Ranjith Kumar C. ${ }^{1}$, Laxmikanth $\mathbf{B}^{2, *}$, Srinivas Nagaram ${ }^{3}$ \\ ${ }^{1}$ Assistant Professor, Dept. of Biochemistry, S.V.S Medical College, Mahabubnagar, Telangana, ${ }^{2}$ Associate Professor, \\ ${ }^{3}$ Demonstrator, Dept. of Biochemistry, Maheshwara Medical College, Chitkul, Medak, Telangana, India
}

*Corresponding Author:

E-mail: drbachulaxmikanth@gmail.com

Received: $06^{\text {th }}$ December, 2017

Accepted: $02^{\text {nd }}$ January, 2018

\begin{abstract}
Introduction and Objectives: Thyroid dysfunction and autoimmunity are related to adverse impact on fertility in reproductive aged women. Anti-Mullerian hormone (AMH), biomarker of 'ovarian age' may be affected by impaired thyroid function, but the relationship between AMH and thyroid hormone is unclear. The aim of this study was to determine the impact of thyroid stimulating hormone (TSH) on AMH levels.

Materials and Methods: In this case-control study, to identify the impact of thyroid hormone on ovarian reserve, we recruited 30 consecutive Indian infertile patients and 30 normal fertile women aged 19-39 years without impact factors on thyroid and ovarian functions between April 2017 and September 2017. We assessed patient age, AMH, and TSH levels of all study participants as independent variables. We also evaluated the correlations between AMH and TSH in our study groups. In addition, independent variables were subjected to multiple regression analysis.

Results: Our results showed significant changes in AMH and TSH between groups. The increased TSH status in the subfertile cases was inversely correlated with the TSH levels $(r=-0.44, \mathrm{p}<0.05)$. The TSH levels were independently associated with the decreased AMH levels in infertile patients $(\beta=-0.82 \mathrm{p}=<0.0001)$.

Conclusion: AMH levels were inversely correlated with TSH levels in infertile women of reproductive age. Further studies with large sample size are warranted in future.
\end{abstract}

Keywords Infertility, Hypothyroidism, Thyroid stimulating hormone, Anti-Mullerian hormone, Ovarian reserve.

\section{Introduction}

In women, thyroid dysfunction is the most common endocrine disorder of reproductive age. Normal thyroid function is important to maintain normal reproduction. ${ }^{1}$ Both Overt and subclinical hypothyroidism may cause menstrual irregularities and anovulation and has been associated with female infertility. ${ }^{2}$ Also, both hyperthyroidism and hypothyroidism are known to be associated with the menstrual disturbance, anovulatory cycles and decreased fecundity. ${ }^{3}$ Menstrual irregularities and anovulation are commonly present in Overt hypothyroid women because of altered peripheral estrogen metabolism, hyperprolactinemia and abnormal release of gonadotropin-releasing hormone. ${ }^{1}$ The prevalence of subclinical hypothyroidism with elevated thyroid stimulating hormone (TSH) and normal freethyroxine (FT4) in infertile women has been reported to be approximately $20 \%$ and is a primary cause of subfertility., ${ }^{4,5}$ TSH is known to be a significant predictor of fertilization failure in women undergoing in vitro fertilization. Therefore, TSH may be a factor influencing ovarian reserve in infertile patients. ${ }^{6}$ The reproductive aging process is thought to be dominated by a gradual decrease in both the quantity and the quality of the oocytes in the ovarian cortex. It is clear that monthly fecundity gradually decreases from the mean age of 30 year onward. ${ }^{7}$ Anti mullerian hormone
(AMH), also known as Mullerian inhibiting substance is a dimeric glycoprotein produced by granulosa cells from pre-antral and antral follicles. The main role of $\mathrm{AMH}$ is to inhibit follicular development from primordial to primary follicular stages. ${ }^{8}$ Serum AMH levels decrease invariably and are undetectable near menopause. ${ }^{7}$ Increasing age in females associated with decrease in fecundity, primarily because of decreased ovarian function. Serum AMH levels were closely correlated with the number of primordial follicles; therefore, AMH is a suitable biomarker of ovarian age in women of reproductive age. ${ }^{9}$ Ovarian function may be affected by impaired thyroid function. Based on this background, our aim of this study was to determine the impact of thyroid stimulating hormone on AMH levels and to report any associations between them in women of reproductive age.

\section{Materials and Methods}

The present study was carried out between April 2017 and September 2017. Cases were chosen from patients who visited the fertility outpatient clinic at the department of Obstetrics and Gynaecology of S.V.S Medical and Hospital and were diagnosed as infertile according to the previously established criteria were recruited into this study. ${ }^{6}$ We also consecutively recruited 30 age matched normal fertile women aged 21 - 35 years. They had no history of treatment for 
infertility or thyroid disorders. We measured TSH and serum AMH levels in the cases and control women. We included newly diagnosed thyroid abnormality, but excluded the patients being treated for thyroid dysfunction. Only women with normal TSH levels (0.4 $-4.5 \mu \mathrm{IU} / \mathrm{mL}$ ) were eligible for enrolment. Informed consent was obtained from all study participants and the study protocol was approved by the institutional ethics committee. Infertility is defined as the failure to achieve clinical pregnancy after 12 months or more. Serum levels of luteinizing hormone, follicle stimulating hormone, estradiol, progesterone, prolactin and testosterone were analysed at 2-5 days of menstrual cycle to screen for infertile patients. The exclusion criteria for this study have been presented in Table 1 .

To investigate the relationship between thyroid function and serum AMH levels, we matched patients by age as confounding factor using 1:1 matching for statistical analysis of healthy fertile women and infertile patients. Blood samples were collected from all study participants, and serum TSH was measured using a commercial electrochemiluminescence immunoassay (Siemens centaur CP). Serum AMH levels were measured in CLIA based CLIMAX instrument by using Acculite reagents.

The data obtained were presented as means and standard deviations. Data comparisons between grouos were done using student's t test or the Mann-Whitney $\mathrm{U}$ - test, as appropriate. Independent variables such as patient AMH and TSH levels which strongly correlated with infertility were estimated by stepwise logistic regression analysis. Finally, Independent variables that were strongly correlated with an infertility related variable were estimated by multiple regression analysis. A $p$ - value of $<0.05$ was considered statistically significant. All statistical analyses were performed using the $\mathrm{R}$ version 3.2.3 with $\mathrm{R}$ commander.

\section{Results}

As presented in Table 2, our study groups were matched for sample size and age. The AMH levels were significantly decreased in the subfertility group when compare to normal controls ( $\mathrm{p}$ value $<0.05$ ). Whereas, the serum TSH was found to be significantly increased in the patient group as compared to that of age matched controls. As depicted in Fig. 1, AMH was inversely correlated with TSH status in our subfertile group ( $r=-$ $0.44, p<0.05$ ) while there was no significant correlation between them in our fertile group. However, there was no significant association of age with AMH levels indicating that TSH influence ovarian reserve and AMH levels in subfertility group was independent of age. Multiple regression analysis showed that TSH levels has a significant influence on the AMH levels in infertile patients (TSH levels standardized partial regression coefficient $(\beta),-0.2 ; p=<0.05)$. Whereas, in the fertile women serving as our control group, age showed a significant impact on AMH levels $(\beta=-0.41$; $\mathrm{p}=<0.001$ ).

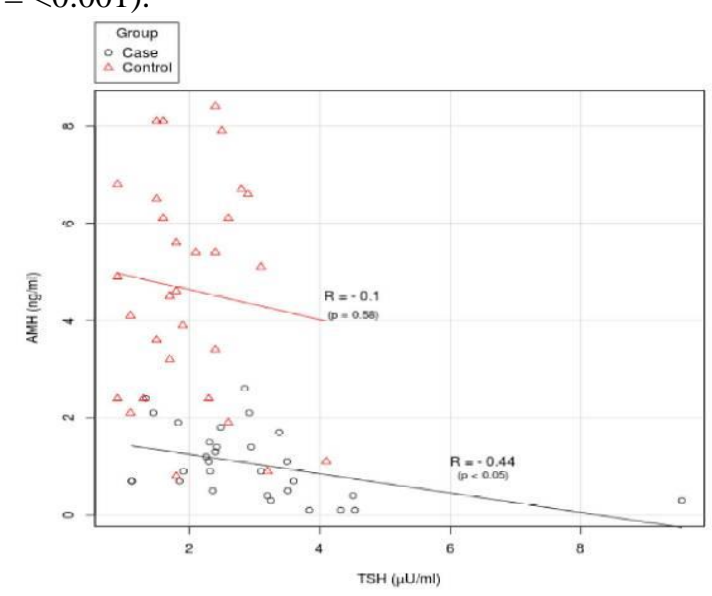

Fig. 1

Table 1: The criteria for exclusion applied in this study

\begin{tabular}{|l|}
\hline Exclusion Criteria/Reasons for exclusion \\
\hline Primary ovarian insufficiency \\
\hline Premature ovarian failure \\
\hline Treated thyroid dysfunction \\
\hline Endometriosis \\
\hline Ovarian tumor \\
\hline Post ovarian surgery \\
\hline Smoking \\
\hline Alcoholism \\
\hline
\end{tabular}

Table 2: Characteristics of women with Fertility (Control) and Subfertility (Cases) groups

\begin{tabular}{|l|c|c|c|}
\hline Variable & Fertility & Subfertility & P - Value \\
\hline Sample size & 30 & 30 & - \\
\hline Age $(\mathrm{yrs})$ & $27.2 \pm 4.46$ & $28.03 \pm 4.07$ & $\mathrm{NS}$ \\
\hline $\begin{array}{l}\text { AMH } \\
(\mathrm{ng} / \mathrm{ml})\end{array}$ & $4.63 \pm 2.21$ & $1.06 \pm 0.69$ & $<0.05$ \\
\hline TSH $(\mu)$ & $2 \pm 0.75$ & $2.94 \pm 1.53$ & $<0.05$ \\
\hline
\end{tabular}

AMH: Anti mullerian hormone, TSH: Thyroid stimulating hormone, NS: Statistically not significant

\section{Discussion}

The results of this study showed a significant decrease in serum AMH levels in infertile group as compared to the controls. This decrease in AMH was inversely correlated with the TSH status in the patient group ( $p<0.05)$. The serum TSH values of case group is significantly higher compared to control group ( $\mathrm{p}<$ 0.05 ) indicating higher TSH values have impact on serum AMH levels as well as primordial follicle count in case group in compare with fertility group. This study findings are well in line with the previous studies on relation between AMH and primordial follicle count. ${ }^{69}$ These studies revealed an inverse correlation between TSH and AMH levels in infertile women with decreased ovarian function without other factors 
affecting thyroid and ovarian function. ${ }^{69}$ Cramer et al showed that TSH acts as predictor of fertilization failure in women undergoing IVF. ${ }^{10}$ Therefore, TSH may be an important factor influencing ovarian reserve in infertile patients. The present study also supports the previous findings by Cramer et al. ${ }^{10}$ Furthermore, in one study, the role of TSH in implantation and embryo development during early pregnancy has been well documented. ${ }^{11}$

Therefore, it is clear that subclinical hypothyroid women with high TSH values are at increased risk for infertility. Clinical evidence suggests a diminished ovarian reserve in infertile patients with elevated serumTSH levels. ${ }^{12}$ A study related to drug induced hypothyroidism in rats proved the similar result. ${ }^{(13)}$ Some previous animal studies proved the role of TSH in follicular development. ${ }^{14,15}$ Mehendale et al reported the follicle development suppression by TSH in a concentration dependent basis. ${ }^{16}$

Though our study findings are well in support of previous findings, it has got some limitations. Firstly, it was a single center observational study. Secondly, limited number of cases and controls are included. Finally, the study has not included the role of autoimmune thyroid antibodies involvement. Therefore, further studies with large sample size at multiple centers are warranted.

\section{Conclusion}

In conclusion, we showed an inverse relationship between serum TSH and AMH levels in infertile women of reproductive age. These findings may influence current infertility treatment strategies and importantly highlight the importance of proper thyroid management in women of reproductive age.

\section{References}

1. Krassas GE, Poppe K, Glinoer D. "Thyroid function and human reproductive health" Endocr Rev (2010) Oct; 31(5):702-55.

2. Weghofer A, Barad DH, Darmon S, Kushnir VA, Gleicher N. "What affects functional ovarian reserve, thyroid function or thyroid autoimmunity?" Reprod Biol Endocrinol (2016) May 10;14(1):26.

3. Elahi S, Tasneem A, Nazir I, Nagra SA, Hyder SW. "Thyroid dysfunction in infertile women" J Coll Physicians Surg Pak (2007) Apr;17(4):191-4.

4. De Groot L, Abalovich M, Alexander EK, Amino N, Barbour L, Cobin RH, Eastman CJ, Lazarus JH, Luton D, Mandel SJ, Mestman J, Rovet J, Sullivan S.

"Management of thyroid dysfunction during pregnancy and postpartum:an Endocrine Society clinical practice guideline" J Clin Endocrinol Metab (2012) Aug;97(8):2543-65.

5. van den Boogaard E, Vissenberg R, Land JA, van Wely M, Ven der Post JA, Goddin M, Bisschop PH.

"Significance of (sub) clinical thyroid dysfunction and thyroid autoimmunity before conception and in early pregnancy: a systemic review Hum Repord Update" (2016) Jun;22(4):532-3.

6. Kuroda K, Uchida T, Nagai S, Ozaki R, Yamaguchi T, Sato Y, Brosens JJ, Takeda S. "Elevated serum thyroidstimulating hormone is associated with decreased anti Müllerian hormone in infertile women of reproductive age" J Assist Reprod Genet (2015) Feb;32(2):243-7.

7. Broekmans FJ, Soules MR, Fauser BC. "Ovarian aging: mechanisms and clinical consequences" Endocr Rev (2009) Aug;30(5):465-93.

8. Murto T, Bjuresten K, Landgren BM, Stavreus-Evers A. "Predictive value of hormonal parameters for live birth in women with unexplained infertility and male infertility" Reprod Biol Endocrinol (2013) Jul 11;11:61.

9. Hansen KR, Hodnett GM, Knowlton N, Craig LB. "Correlation of ovarian reserve tests with histologically determined primordial follicle number" Fertil Steril (2011) Jan;95(1):170-5.

10. Cramer DW, Sluss PM, Powers RD, McShane P, Ginsburgs ES, Hornstein MD, Vitonis AF, Barbieri RL. "Serum Prolactin and TSH in vitro fertilization population:is there is a link between fertilization and thyroid function?" J Assist Reprod Genet (2003) Jun;20(6):210-5.

11. Colicchia M, Campagnolo L, Baldini E, Ulisse S, Valensise H, Moretti C. "Molecular basis of thyrotropin and thyroidhormone action during implantation and early development" Hum Reprod Update (2014) NovDec;20(6):884-904.

12. Michalakis KG, Mesen TB, Brayboy LM, Yu B, Richter KS, Levy M, Widra E, Segars JH. "Subclinical elevations of thyroid-stimulating hormone and assisted reproductive technology outcomes" Fertil Steril (2011) Jun 30;95(8):2634-7.

13. Dijkstra G, de Rooij DG, de Jong FH, van den Hurk R. "Effect of hypothyroidism on ovarian follicular development, granulosa cell proliferation and peripheral hormone levels in prepubertal rat" Eur J Endocrinol (1996) May;134(5):649-54.

14. Fedail JS, Zheng K, Wei Q, Kong L, Shi F. "Roles of thyroid hormones in follicular development in the ovary of neonatal and immature rats" Endocrine (2014) Aug;46(3):594-604.

15. Zhang C, Wang X, Wang Z, Niu W, Zhu B, Xia G. "Effect of different culture systems and 3,5, 3triiodothyronine/follicle- stimulating hormone on prenatal follicle development in mice" PLoS One (2013) Apr 15;8(4):e61947.

16. Mehendale RG, Bruot BC. "Thyroid stimulating hormone inhibits rat granulosa cell steroidogenesis in primary culture" Endocrine (1995) Mar;3(3):215-20. 